\title{
Real-world impact of education: treating patients with ipilimumab in a community practice setting
}

\author{
This article was published in the following Dove Press journal: \\ Cancer Management and Research \\ 21 December 2013 \\ Number of times this article has been viewed
}

\author{
Blanca Ledezma' \\ Annie Heng ${ }^{2}$ \\ 'Department of Hematology and \\ Oncology, University of California, \\ Los Angeles, Santa Monica, CA, USA; \\ ${ }^{2}$ Angeles Clinic and Research Institute, \\ Los Angeles, CA, USA
}

\begin{abstract}
After decades without promising new treatments for advanced and metastatic melanoma, ipilimumab was the first systemic therapy approved for use in this patient population. A fully human monoclonal antibody that blocks cytotoxic T-lymphocyte antigen 4 (CTLA-4) to augment antitumor T-cell responses, ipilimumab significantly extended overall survival in clinical trials. Because ipilimumab is associated with a set of immune-related adverse events that likely reflect the agent's mechanism of action, a management guide has been established. Nurses play a significant role in initially identifying these adverse reactions and assisting in patient education, treatment, and follow-up. Herein, we discuss commonly asked questions related to ipilimumab therapy and treatment of adverse events, and how nurses can be prepared to answer these questions as they arise from patients and caregivers.
\end{abstract}

Keywords: melanoma, immunotherapy, patient education

\section{Introduction}

For patients diagnosed with unresectable stage III or IV (advanced) melanoma, historical benchmark data from a meta-analysis estimate a 25\% 1-year survival rate that falls to approximately $15 \%$ by 5 years. ${ }^{1,2}$ Fortunately, several promising new agents have been US Food and Drug Administration (FDA)-approved for treatment of advanced or metastatic melanoma in recent years, or are late in clinical development. Among these new options is ipilimumab, a fully human monoclonal antibody that blocks cytotoxic T-lymphocyte antigen 4 (CTLA-4) to augment antitumor T-cell responses. ${ }^{3,4}$ Ipilimumab is FDA-approved in treatment-naïve and previously treated disease $^{5}$ on the basis of improved overall survival (OS) in two Phase III studies. Importantly, 19\%-36\% of ipilimumab-treated patients were still alive 4 years after study enrollment. These data suggest that ipilimumab provides an unprecedented extension of life in some patients who until recently had few effective options with manageable safety profiles. ${ }^{2,4,6}$

Associated with ipilimumab are a set of treatment-related adverse events that are commonly referred to as immune-related adverse events (irAEs) because they are most likely tied to the agent's immune-related mechanism of action..$^{3,4,7-9}$ In clinical trials, most of these irAEs were mild to moderate, and most were reversible using a set of treatment guidelines that were developed for ipilimumab based on clinical experience with the drug. These guidelines emphasize vigilant follow-up and early use of corticosteroids when appropriate. Rarely, however, some irAEs can be severe, life-threatening, or irreversible despite immunosuppressive therapy., ${ }^{4,7}$
Correspondence: Blanca Ledezma Department of Hematology and Oncology, University of California Los Angeles, 2020 Santa Monica Boulevard - Suite 600, Santa Monica, CA 90404, USA

$\mathrm{Tel}+\mathrm{I} 3109471888$

Email bledezmanp@gmail.com 
Nurses are often the first and most frequent point of contact for patients undergoing cancer treatment. It is therefore crucial that the full clinical management team, particularly nurses, is armed with all necessary information regarding management of patients, side effects, methods of infusion, and other critical aspects related to treatment. Since ipilimumab is a relatively novel treatment with a clinical profile that differs in some respects from those of traditional melanoma therapies, such as cytotoxics, education of this nature related to ipilimumab is particularly important and timely to provide to nurses. Therefore, the purpose of this review is to convey collective learning from ipilimumab clinical trials, case studies, and our own clinical experience to address commonly asked questions related to ipilimumab therapy. These questions include understanding the mechanism of action, efficacy, patient evaluation and follow-up, toxicity management, and patterns of response. We often hear questions on these topics from fellow nurses, but they may also originate from patients and caregivers.

\section{Frequently asked questions: mechanism of action}

The mechanism of action of ipilimumab differs from those of traditional chemotherapy or small-molecule inhibitors, which means that response kinetics may differ as well. ${ }^{10,11}$ Activation of the immune system begins when a T-cell receptor recognizes and binds a foreign compound, or antigen, that is presented on the surface of an antigen-presenting cell. This recognition generates an activation signal to the T-cell. To reinforce this initial activation signal, a costimulatory signal is then provided from the antigen-presenting cell (via the B7 family of molecules) to the T-cell (via the $\mathrm{CD} 28$ receptor). Conversely, to keep the activation signal in check and prevent overstimulation, the T-cell expresses a second receptor, CTLA-4, which also binds B7, but results in inhibition of the T-cell. The balance of these stimulatory and inhibitory signals determines whether the T-cell. is activated in response to the antigen or fails to respond (anergy) (Figure 1A). Preclinical and clinical research have revealed that in many types of cancers, tumors evade elimination by the immune system by tipping the balance toward anergy using a variety of mechanisms, some of which directly involve CTLA-4. ${ }^{12}$ Thus, monoclonal antibodies that bind CTLA-4 were developed as anticancer therapies under the theory that through blockade of the CTLA-4-mediated inhibitory signal, the activity of T-cells may be activated against tumor antigens and their activity harnessed for treatment of cancer. ${ }^{3,13}$
A



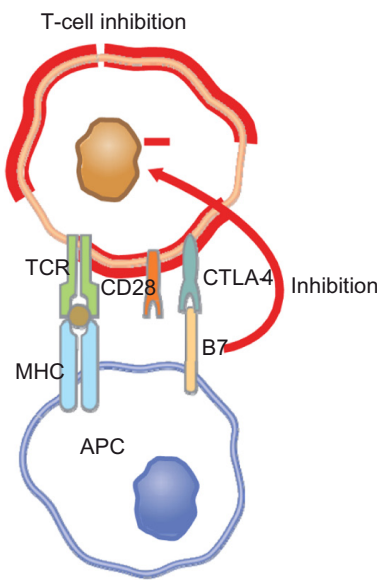

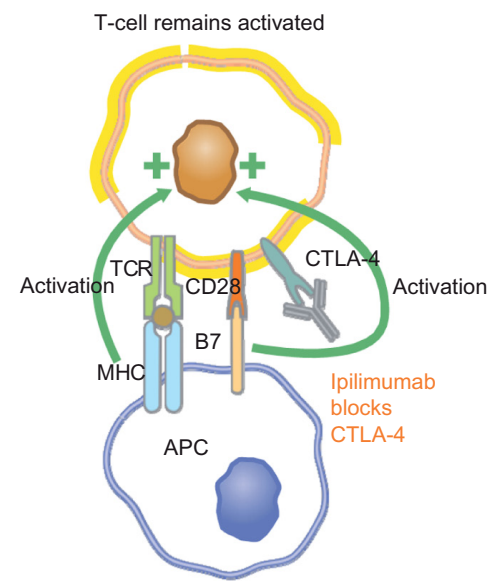

B

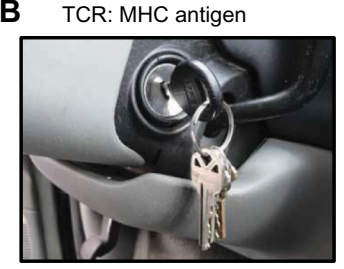

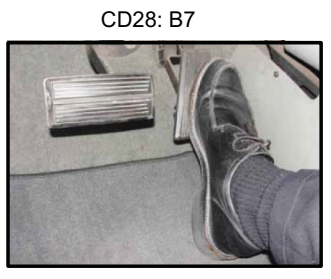

T-cell activation

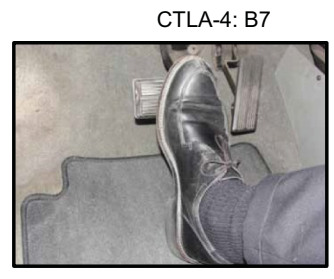

T-cell inhibition

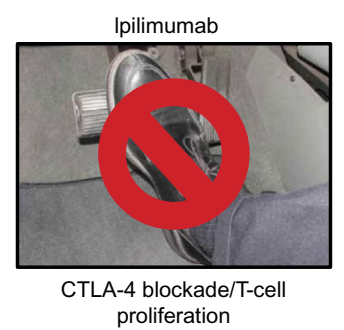

Figure I (A and B) Role of CTLA-4 in T-cell responses and the impact of CTLA-4 blockade with ipilimumab. Ipilimumab mechanism of action (A) and "brake and pedal" analogy (B) as used to explain the mechanism to patients and caregivers.

Abbreviations: CTLA, cytotoxic T-lymphocyte antigen; APC, antigen-presenting cell; MHC, major histocompatibility complex; TCR, T-cell receptor. 
We are often asked by other nurses how to explain these challenging immunologic concepts and the mechanism of action of ipilimumab in terms that patients understand. One analogy we employ is to equate the patient's immune system to a car (Figure 1B). ${ }^{14}$ Depressing the accelerator (equivalent to activating T-cells) is necessary for the car to move forward (equivalent to a productive immune response against the tumor). Further, just as a car has a brake to keep forward motion in check, the body's natural response to immune activation is to keep the immune response in check via engagement of inhibitory pathways, such as CTLA-4. Ipilimumab, by binding CTLA-4, is functioning to "lift the foot off the brake" so that the car can continue forward, ie, so that a T-cell-mediated antitumor immune response can continue. We find that through this analogy, patients can more easily understand the drug's mechanism of action and are often encouraged by the idea that their own body is fighting their cancer. In addition, a basic understanding of how ipilimumab modulates the immune response makes it easier for patients to understand the risks of adverse events associated with ipilimumab, and it sets patient expectations about how rapidly they may respond to treatment. These topics are covered below.

\section{Frequently asked questions: dose and schedule Dose adjustments}

The approved dose of ipilimumab is $3 \mathrm{mg} / \mathrm{kg}$, administered intravenously over 90 minutes every 3 weeks for a total of four doses. ${ }^{5}$ Fellow nurses or other health care professionals frequently inquire whether dose adjustments can be made. Of note, a higher dose of ipilimumab $(10 \mathrm{mg} / \mathrm{kg})$ is currently being studied, but is not FDA approved; in addition, lower doses have been evaluated as well. A Phase II dose-ranging study did show that the frequency of irAEs of any grade did increase with ipilimumab dose, and a Phase III study comparing the $3 \mathrm{mg} / \mathrm{kg}$ with the $10 \mathrm{mg} / \mathrm{kg}$ dose is ongoing. ${ }^{15,16}$ At present, therefore, use at doses other than $3 \mathrm{mg} / \mathrm{kg}$ is not recommended outside a clinical trial setting. To mitigate irAEs, the US prescribing information Risk Evaluation and Mitigation Strategy (REMS) for ipilimumab provides suggestions for when to withhold (Table 1) or permanently discontinue (Table 2) ipilimumab therapy based on the occurrence and severity of irAEs. ${ }^{9}$ Furthermore, no dose adjustment of ipilimumab is required or routinely made for body weight after administration on a $\mathrm{mg} / \mathrm{kg}$ basis, ${ }^{5}$ and actual (not ideal) weight is used. The exception, as aligned with most institutional guidelines for cancer therapies, is made when a patient experiences a significant weight gain or loss during the course of therapy, and the dose may be recalculated in those instances.
Table I When to withhold ipilimumab

\begin{tabular}{ll}
\hline Organ system & $\begin{array}{l}\text { Withhold if patient experiences one of the } \\
\text { following }\end{array}$ \\
\hline Gastrointestinal & - Four to six stools per day over baseline or more \\
& - Abdominal pain \\
& - Blood or mucus in stool \\
- & Moderate-to-severe signs of patient-reported \\
Skin & rash or pruritus \\
& - Diffuse rash over less than $50 \%$ of skin surface \\
Liver & - AST or ALT $>2.5$ to $\leq 5.0 \times$ ULN \\
Neurologic & - Moderate symptoms, including muscle weakness, \\
& motor or sensory neuropathy, but with no impact \\
& on activities of daily living \\
Endocrine & Signs or symptoms of dysfunction, including: \\
& fatigue, headache, mental status changes, \\
& abdominal pain, hypotension
\end{tabular}

Note: Ipilimumab [US prescribing information]: risk evaluation and mitigation strategy (REMS). Bristol-Myers Squibb. 2012. Available from: http://www.yervoy. com/hcp/rems.aspx. Accessed July 12, 2013.9

Abbreviations: AST, aspartate aminotransferase; ALT, alanine aminotransferase; ULN, upper limit of normal.

\section{Retreatment}

As per the approved indication, ipilimumab is given as four doses every 3 weeks; this 12 -week time frame was termed the

Table 2 When to permanently discontinue ipilimumab

\begin{tabular}{|c|c|}
\hline Organ system & $\begin{array}{l}\text { Permanently discontinue if patient } \\
\text { experiences one of the following }\end{array}$ \\
\hline Gastrointestinal & $\begin{array}{l}\text { - Seven or more stools per day over baseline } \\
\text { - Severe or life-threatening enterocolitis } \\
\text { - Signs of bowl perforation } \\
\text { - lleus } \\
\text { - Fever }\end{array}$ \\
\hline Skin & $\begin{array}{l}\text { - Life-threatening dermatitis, including generalized } \\
\text { exfoliate, full-thickness dermal ulceration, ulcerative } \\
\text { or bullous dermatitis, skin necrosis, Stevens- } \\
\text { Johnson syndrome, toxic epidermal necrolysis }\end{array}$ \\
\hline Liver & $\begin{array}{l}\text { - } \text { AST or ALT }>5.0 \times \text { ULN } \\
\text { - and/or total bilirubin }>3.0 \times \text { ULN }\end{array}$ \\
\hline Neurologic & $\begin{array}{l}\text { - Severe symptoms, including muscle weakness or } \\
\text { sensory alterations that impact on activities of } \\
\text { daily living }\end{array}$ \\
\hline General & $\begin{array}{l}\text { - Failure to complete full treatment course of } \\
\text { ipilimumab within } 16 \text { weeks of first administered } \\
\text { dose } \\
\text { - Inability to taper corticosteroid dose to } 7.5 \mathrm{mg} \\
\text { prednisone per day without symptoms reemerging } \\
\text { - Hospitalizations for any severe or life-threatening } \\
\text { immune-related reaction }\end{array}$ \\
\hline
\end{tabular}

Note: Ipilimumab [US prescribing information]: risk evaluation and mitigation strategy (REMS). Bristol-Myers Squibb. 2012. Available from: http://www.yervoy. com/hcp/rems.aspx. Accessed July 12, 2013.9

Abbreviations: AST, aspartate aminotransferase; ALT, alanine aminotransferase; ULN, upper limit of normal. 
"induction phase" in clinical studies. Following the induction phase, retreatment for qualifying patients was included in the protocols of some ipilimumab Phase II and III clinical trials. The rationale for these inclusions was based on the agent's immune-related mechanism of action, ie, patients who initially experienced clinical benefit might experience reactivation of the immune system in response to further therapy, which may result in recognition and response to residual tumor cells. ${ }^{17}$ In the Phase III registration trial, a subanalysis of patients that received retreatment upon disease progression showed ipilimumab further augmented durable objective responses and/or stable disease. ${ }^{18}$ The safety profile observed during retreatment was similar to that observed during the induction phase of treatment.

The FDA does not contraindicate retreatment in the approved prescribing information, and the National Comprehensive Cancer Network guidelines state that retreatment with ipilimumab may be considered for patients who experienced no significant systemic toxicity during prior ipilimumab therapy, and who relapse after initial clinical response or progress after stable disease for more than 3 months. ${ }^{19}$ Ongoing research will help to further optimize the schedule for ipilimumab administration. ${ }^{20}$

\section{Frequently asked questions: efficacy and response}

An extensive clinical trial program has established ipilimumab efficacy in a percentage of patients with advanced or metastatic melanoma. The registration trial design included an active control group $(n=136)$ of patients who received gp100 vaccine alone and compared it to those randomized to receive ipilimumab alone $(n=137)$ or ipilimumab combined with the active control gp100 $(n=403) .{ }^{4}$ Median OS was significantly greater with the ipilimumab-gp100 combination compared with gp100 alone (10.0 months versus [vs] 6.4 months, hazard ratio [HR] $0.68 ; P<0.001)$. Median OS was also significantly greater with ipilimumab alone compared with gp100 alone (10.1 months vs 6.4 months, HR $0.66 ; P=0.003$ ). One and 2 -year OS rates for ipilimumab alone were $45.6 \%$ and $23.5 \%$, respectively; for gp 100 alone, $25.3 \%$ and $13.7 \%$, respectively; and for ipilimumab plus gp $100,43.6 \%$ and $21.6 \%$, respectively. ${ }^{4}$

In a second Phase III study at the higher dose $(10 \mathrm{mg} / \mathrm{kg})$, OS was significantly longer for ipilimumab in combination with dacarbazine compared with dacarbazine plus placebo (11.2 months vs 9.1, respectively). ${ }^{7}$ OS rates at 1,2 , and 3 years were also significantly higher for ipilimumab plus dacarbazine: these rates were $47.3 \%, 28.5 \%$, and $20.8 \%$, respectively; for placebo plus dacarbazine, they were $36.3 \%$, $17.9 \%$, and $12.2 \%$, respectively. Survival data is also now available for 5 years postenrollment for patients in the Phase II studies of ipilimumab in advanced melanoma, further supporting the idea that ipilimumab provides a substantial long-term OS potential for some patients. ${ }^{21}$ To reiterate, ipilimumab has been evaluated at $10 \mathrm{mg} / \mathrm{kg}$ and in combination with a variety of chemotherapies, targeted agents, and other immunotherapies, but those doses and schedules have not yet been approved by the FDA for use in patients.

Immunotherapies such as ipilimumab stimulate the patient's immune system to mount an endogenous antitumor immune response (Figure 1). ${ }^{22}$ Because of this immunerelated mechanism of action, responses to ipilimumab may differ from patient to patient, and it often takes a certain amount of time for patients to mount a response. ${ }^{23}$ Among patients with metastatic melanoma who experienced a positive outcome from ipilimumab therapy in clinical trials, four patterns of response were observed (Figure 2). ${ }^{23}$ As with chemotherapy, patients treated with immunotherapy may demonstrate an immediate reduction in baseline lesions without the presence of new lesions. Ipilimumab has also yielded durable stable disease in some patients, and in some cases it has been followed by a slow, steady decline in total tumor burden. Some ipilimumab-treated patients may present with new lesions concurrently with a decrease in overall burden of disease. Occasionally, a positive tumor response has been observed in patients following an initial increase in total tumor burden. ${ }^{23,24}$ The idea that a tumor response is possible despite the appearance of new lesions or an initial increase in tumor burden may be unfamiliar to the medical team, and may be of concern to patients, if they are not prepared for this possibility.

We are frequently asked by our nurse colleagues how to determine the difference between delayed response and progression. In our practices, we base therapeutic decisions on multiple factors that include patient labs and overall clinical performance. If following ipilimumab therapy, a patient exhibits radiographic progression but their labs are stable, especially lactate dehydrogenase (LDH), and they generally feel well or feel they are improving, we may hold to monitor for delayed response with another scan in about 4-6 weeks. Conversely, if the labs are worsening, LDH is increasing, and the patient is physically deteriorating, then nurses note the suspected progression, deferring to the treating physician to make further therapeutic decisions.

This decision-making process related to ipilimumab differs from the typical experience with chemotherapy or 


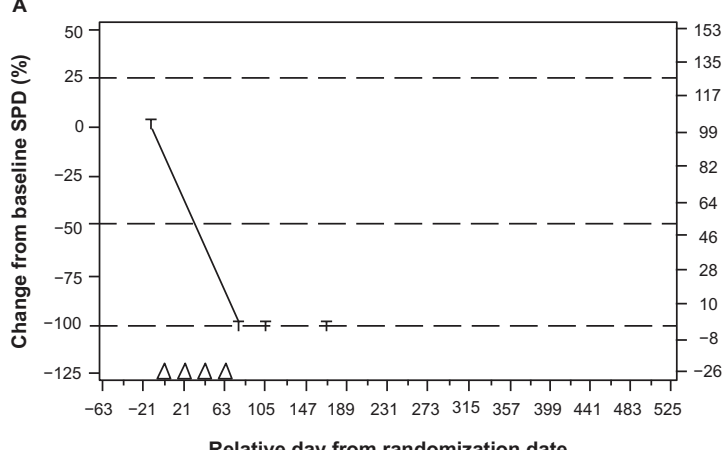

C

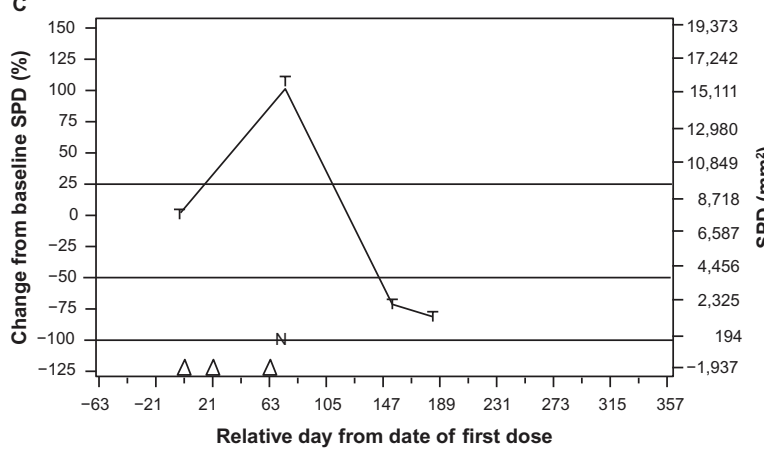

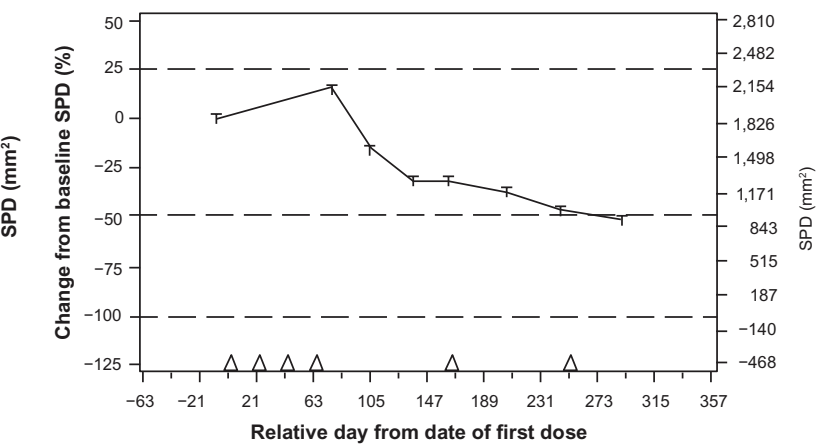

D

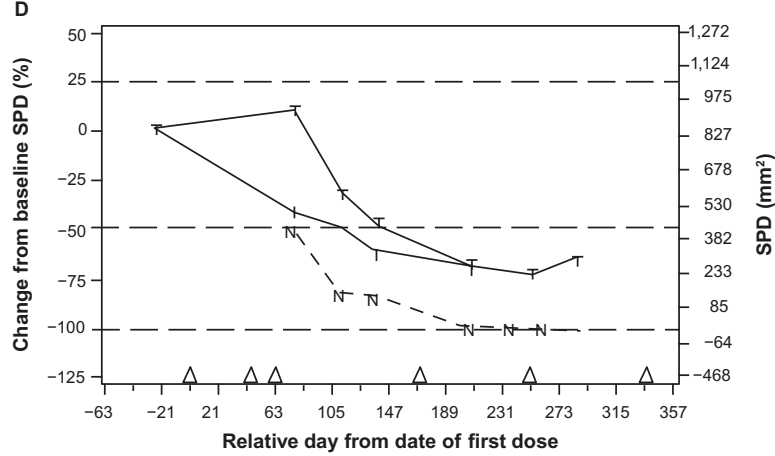

Figure 2 (A-D) Established patterns of response to ipilimumab.

Notes: Four patterns of response to ipilimumab include (A) response in baseline lesions, (B) stable disease, (C) response after initial increase in total tumor volume, and (D) reduction in total tumor burden after the appearance of new lesions. All four patterns of response have been observed in patients who have been treated with ipilimumab, and all have been associated with positive outcomes in patients. N, tumor burden of new lesions (C and $\mathbf{D}$ ). (D) top line, total tumor burden; middle line, tumor burden of baseline lesions; bottom line, tumor burden of new lesions. Triangles, ipilimumab dosing time points; dashed lines, thresholds for response or PD/irPD. Reprinted from Clin Cancer Res, 2009, 15(23), 74I2-7420, Wolchok JD, Hoos A, O'Day S, et al, Guidelines for the evaluation of immune therapy activity in solid tumors: immune-related response criteria, ${ }^{23}$ with permission from the American Association for Cancer Research (AACR). Copyright @ 2009.

Abbreviations: irPD, immune-related progressive disease; SPD, sum of the product of perpendicular diameters.

targeted therapy. In fact, because beneficial responses may be atypical, the most appropriate end point to evaluate responses to ipilimumab is still a subject of debate across the oncology community. ${ }^{23,24}$ At present, however, nurses need to understand the interrelatedness of ipilimumab's mechanism of action and efficacy, and the symptoms that the patient may experience during therapy, and be prepared to answer questions from patients and caregivers. For example, palpable tumors may become more painful; change in color, warmth, or tenderness; or increase in size during treatment with ipilimumab. When patients report these phenomena, we explain that they could be related to a T-cell-mediated attack on the tumors, and that this will be confirmed through continued monitoring for a subsequent decrease in tumor size or other response. In short, we counsel patients and caregivers on the immunologic mechanism of action of ipilimumab as a method of helping to explain treatment-related changes that patients may experience. Such counsel also appears to relieve some concerns around why immediate tumor regression may not occur in every patient who receives therapy. It also supports the rationale for waiting until week 12 for the initial scan as per guidelines, or possibly longer in some patients, to allow time for the immune system to mount a response to tumors.

\section{Frequently asked questions: identification and management of ipilimumab-associated toxicities Kinetics of onset and resolution of irAEs}

As already noted, most adverse reactions to ipilimumab are inflammatory in nature. ${ }^{4,7-9}$ The most common irAEs affect the skin (rash, pruritus) or gastrointestinal system (GI; diarrhea, nausea, abdominal pain); endocrine, hepatic, and neurologic events occur with less frequency (Table 3), 4,7,25 Individual clinical studies and a pooled analysis across the ipilimumab clinical program showed most irAEs were reported during the induction dosing period. ${ }^{3,4,7,25}$ Median time to onset of irAEs was approximately 5-9 weeks, depending on dose of ipilimumab and organ class affected (Figure 3) ${ }^{8,26}$ Skin reactions seem to appear first, on average within the first couple of weeks of treatment, with GI 
Table 3 Frequency of immune-related adverse events (irAEs) in pooled analysis across Phase I-III ipilimumab clinical trials

\begin{tabular}{llll}
\hline & $\begin{array}{l}\text { Any grade, } \\
\mathbf{n}(\%)\end{array}$ & $\begin{array}{l}\text { Grade 3-4, } \\
\mathbf{n}(\%)\end{array}$ & $\begin{array}{l}\text { Grade 5, } \\
\mathbf{n}(\%)\end{array}$ \\
\hline Any irAEs & $962(64.2)$ & $266(17.8)$ & $9(0.6)$ \\
Dermatologic & $672(44.9)$ & $39(2.6)$ & $0(0)$ \\
Gastrointestinal & $487(32.5)$ & $137(9.1)$ & $3(0.2)$ \\
Endocrine & $68(4.5)$ & $34(2.3)$ & $0(0)$ \\
Hepatic & $24(1.6)$ & $16(1.1)$ & $2(0.1)$ \\
Ocular & $20(1.3)$ & $6(0.4)$ & $0(0)$ \\
Neurologic & $2(0.1)$ & $0(0)$ & $1(<0.1)$ \\
Cardiovascular & $2(0.1)$ & $2(0.1)$ & $0(0)$ \\
\hline Not)
\end{tabular}

Notes: Pooled data across the ipilimumab clinical program shows that most patients experience an irAE of some grade. These events are further broken down by organ class, showing the total number of patients and the percentage of patients (in parentheses) that experienced irAEs of each type. The table further shows how many of these events were serious (grade 3-4) or fatal (grade 5). Note that subjects may have had more than I event. Unknown intensities are included in the "Any grade" column. In the case of cardiovascular events, two cases of myocarditis were reported. Reprinted with permission. (C) 2011 American Society of Clinical Oncology. All rights reserved. Ibrahim RA, Berman DM, de Pril V, et al. Ipilimumab safety profile: summary of findings from completed trials in advanced melanoma. J Clin Oncol. 20I I;29 Suppl:8583.25

reactions following shortly afterward. Endocrinopathies, in contrast, can occur late in the induction treatment phase or even weeks or months following the last dose of ipilimumab. Despite these observed trends, the clinical team should remain vigilant for irAEs throughout treatment and in the weeks or months afterward, and instruct patients to do the same. As part of this vigilance, nurses should continue to follow-up with patients even after they have completed ipilimumab therapy. Events managed as per the treatment guidelines were generally resolved within 4-8 weeks. ${ }^{25,26}$

\section{Education and testing before treatment}

The nursing team plays a critical role in continuously educating patients and caregivers to alleviate fears and misconceptions that may arise before, during, and after treatment. As part of this role, nurses should prepare patients for what to expect and what to look for in terms of signs and symptoms of irAEs, and emphasize that early reporting of possible irAEs and prompt intervention increases the likelihood that the patient can continue to receive the drug on schedule, maximizing the chances that he or she will benefit from therapy.

It is recommended that nurses provide a questionnaire for patients to answer prior to each infusion, or verbally cover a series of questions on signs and symptoms (Figure 4). Information in the responses can be used to identify and help guide discussions between nurses and patient/caregivers. It can often help distinguish between specific irAEs, eg, whether the diarrhea that a patient is experiencing might be associated with a GI or an endocrine toxicity. It can also capture a potential irAE promptly, so that with early intervention, higher-grade adverse events may be prevented.

Important labs at baseline and prior to each infusion include full thyroid-function (thyroid-stimulating hormone, free triiodothyronine and thyroxine, cortisol, adrenocorticotropic hormone, testosterone, luteinizing hormone, follicle-stimulating hormone) and liver-function tests, as recommended by the FDA. ${ }^{5}$ Additionally, testing for LDH levels may help to discern how patients are trending. In some cases, lipase and amylase may be drawn to rule out pancreatitis.

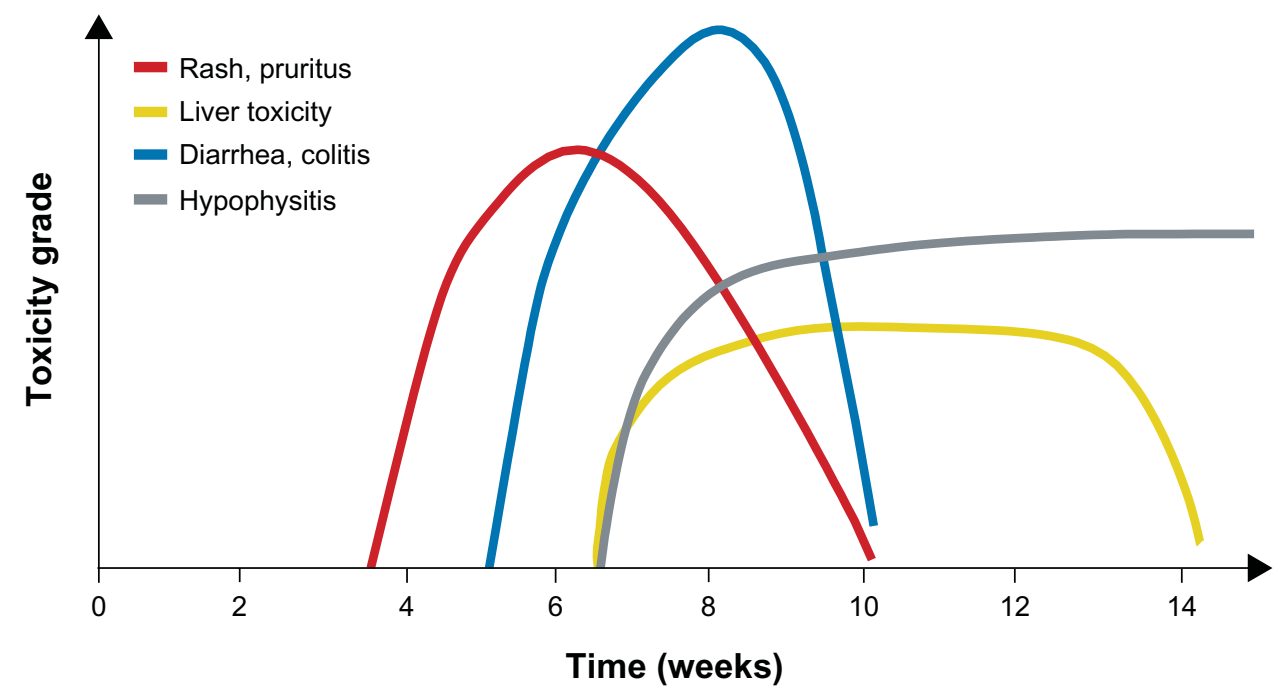

Figure 3 Average time to onset of adverse events associated with ipilimumab. Kinetics of appearance of immune-related adverse events by organ class over time. Note: Reprinted with permission. (C) 2012 American Society of Clinical Oncology. All rights reserved. Weber JS, Kähler KC, Hauschild A. Management of immune-related adverse events and kinetics of response with ipilimumab. J Clin Oncol. 2012;30(21):2691-2697. ${ }^{8}$ 


\section{Ask patients if they are experiencing any of the following symptoms:}

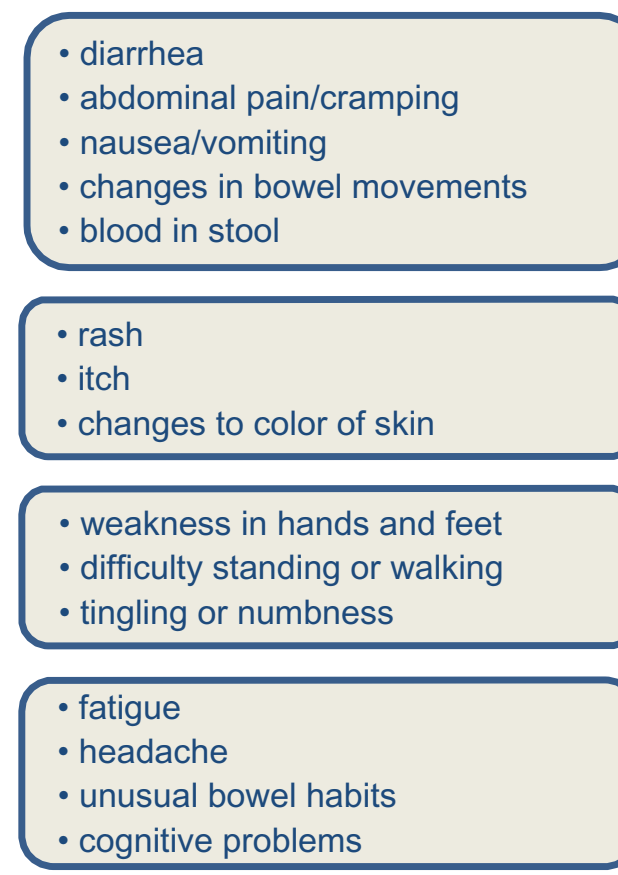

\section{Consider potential toxicity and contact provider:}
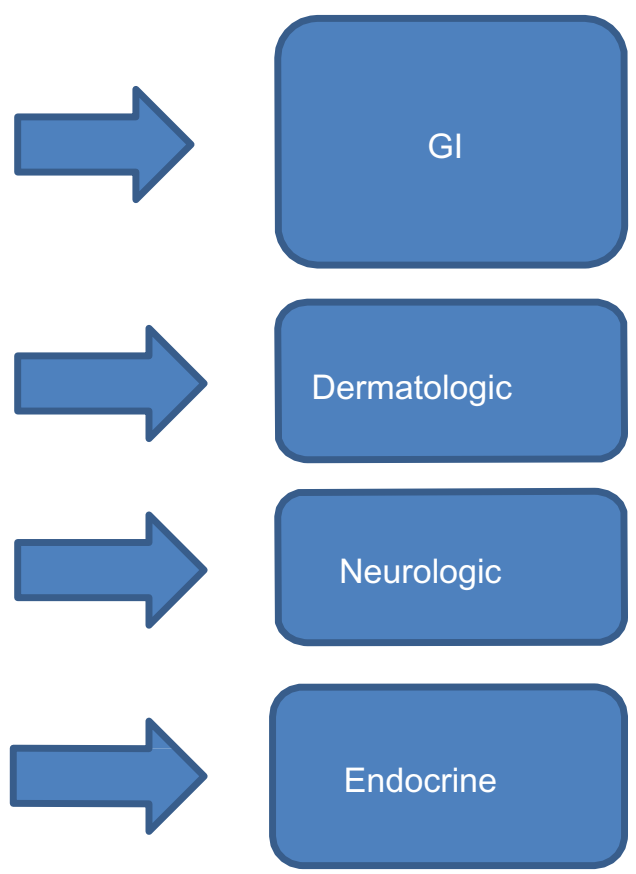

General symptoms that may require follow-up:

fever, vision changes, difficulty sleeping, changes in appetite, difficulty performing daily activities, respiratory distress, pain, coughing

Figure 4 Common signs and symptoms of immune-related adverse events (irAEs). Signs and symptoms nurses should monitor for at every patient visit to assist in early identification of irAEs associated with ipilimumab. Ipilimumab [US prescribing information]: risk evaluation and mitigation strategy (REMS). Bristol-Myers Squibb. 2012. Available from: http://www.yervoy.com/hcp/rems.aspx. Accessed July I2, 2013.9

Abbreviation: $\mathrm{Gl}$, gastrointestinal.

These data will help establish the patient's baseline and later inform whether an antitumor immune reaction is occurring.

\section{Management during and after treatment}

Any clinic administering ipilimumab should have an established management protocol in place to mitigate irAEs, and there is a wealth of information from the ipilimumab REMS and other published clinical trial data to inform the teams. ${ }^{3,4,7,9,25}$ Most low-grade events can be managed symptomatically, eg, creams and oatmeal baths may alleviate very minor skin reactions, and over-the-counter treatments may help manage low-grade diarrhea but should only be taken under medical supervision. Persistent or higher-grade events require prompt intervention with corticosteroid therapy, which should be started as soon as the patient reports highergrade or recurrent problems in accordance with established guidelines in the REMS; ipilimumab may need to be withheld or permanently withdrawn in some circumstances (Tables 1 and 2). ${ }^{9}$ Upon improvement to grade 1 or baseline, corticosteroids should be tapered over at least 1 month. In some patients, referral to an endocrinology or GI specialist may be necessary to assist in the management of certain toxicities. As previously mentioned, nurses have the greatest contact with the patients, and may likely be the ones that recognize the need for referral and inform the physician.

\section{Individualized follow-up and counseling}

To ensure adequate and personalized management takes place, our practice has established a system of daily telephone communication between nurses and the patients experiencing irAEs (Figure 5). We recommend that our colleagues consider implementing similar systems that align 
Step 1: Daily phone calls to the patients experiencing irAEs by nursing staff

- keep track of calls to patients on a whiteboard or other easily visible method

Step 2: Discussion with the patient

- update on symptoms

- reminders of proper management with correct dosage of medication

- question patient about hydration, food changes, discomfort, changes in mood or sleep, and other points relevant to their specific irAE

Step 3: Determine alterations in medication and whether the patient needs to visit the clinic sooner than scheduled

- instruct patients to visit the emergency room (ER) if irAE is high-grade or life-threatening

Step 4:Schedule additional office visits between doses of ipilimumab

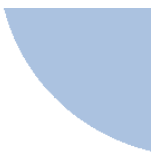

- monitor patients and draw labs

Figure 5 Sample of nurse's role in management protocol when immune-related adverse events (irAEs) occur in patients.

with the needs of their individual practices. Such a system should track the daily patient point of contact in a way that is easily accessible to all (eg, a whiteboard). Updated with this information, the team may recommend increased office visits between doses to monitor patients and draw labs for patients who are experiencing an irAE. We also recommend special preparation for patients traveling out of state or the country (Figure 6). These proactive management steps may prevent escalation of mild irAEs to more serious events that are more likely to require emergency room visits.

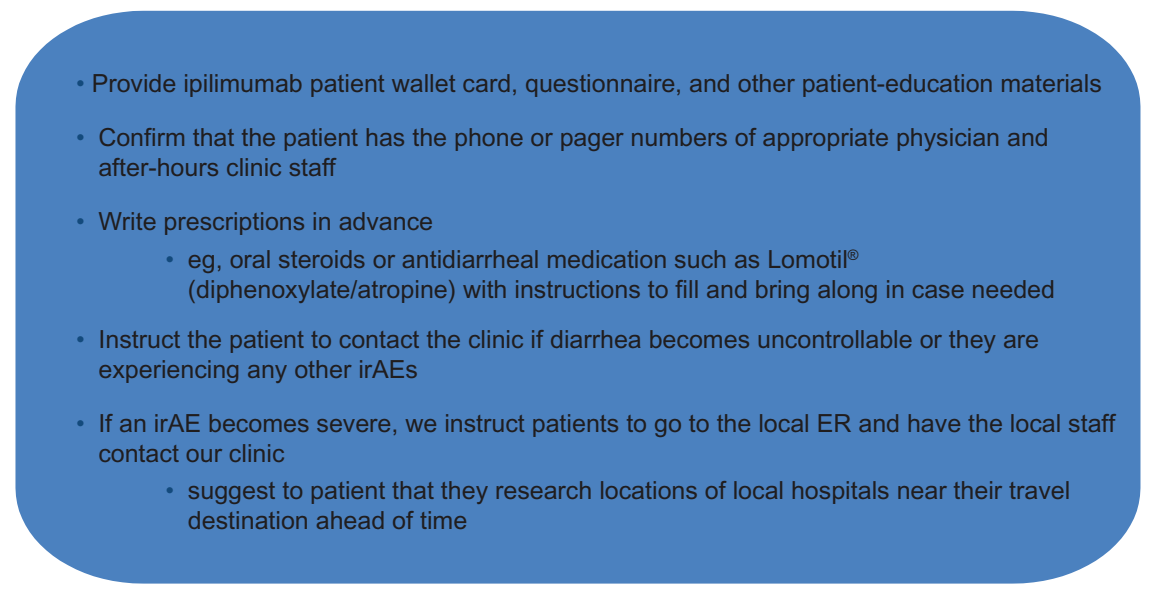

Figure 6 Preparation for patients traveling out of state or country during ipilimumab treatment. Abbreviation: irAE, immune-related adverse event. 
Furthermore, prompt management and resolution of irAEs may allow patients to continue or resume ipilimumab therapy where appropriate.

\section{Discussion}

Ipilimumab has demonstrated the potential to extend life in patients with unresectable or metastatic melanoma, a disease that until recently has had a poor prognosis. Moreover, this agent has shown efficacy in patients with brain metastases and other characteristics associated with poorer prognosis. ${ }^{4}$ Most patients will experience an irAE during their course of treatment with ipilimumab, ${ }^{25}$ and these toxicities require decision-making and management activities that may be somewhat unfamiliar to the health care team. Newer, more effective oncology therapies like ipilimumab are becoming more widely used, and to ensure these therapies meet their full therapeutic potential, it is essential that nurses and other members of the management team be thoroughly educated about appropriate use, patterns of response, safety, and administration. This education will in turn give nurses the knowledge and confidence to educate their patients. Not only does this process help relieve patients' and caregivers' anxieties but it also facilitates timely identification and prompt treatment of irAEs. It is our hope that the information provided in this review will further these efforts and serve as a foundation for broader nursing education programs at hospitals or clinics.

\section{Acknowledgment}

The authors take full responsibility for the content of this publication and confirm that it reflects their viewpoints and medical expertise. The authors wish to acknowledge StemScientific, funded by Bristol-Myers Squibb, for providing writing and editorial support. Neither Bristol-Myers Squibb nor StemScientific influenced the content of the manuscript, nor did the authors receive financial compensation for authoring the manuscript.

\section{Disclosure}

The authors have no conflicts of interest to disclose.

\section{References}

1. Korn EL, Liu PY, Lee SJ, Chapman JA, et al. Meta-analysis of phase II cooperative group trials in metastatic stage IV melanoma to determine progression-free and overall survival benchmarks for future phase II trials. J Clin Oncol. 2008;26(4):527-534.

2. Altekruse SF, Kosary CL, Krapcho M, et al, editors. SEER Cancer Statistics Review, 1975-2007. Bethesda, MD: National Cancer Institute; 2010. Available from: http://seer.cancer.gov/csr/1975_2007. Accessed September 22, 2011.
3. Hoos A, Ibrahim R, Korman A, et al. Development of ipilimumab: contribution to a new paradigm for cancer immunotherapy. Semin Oncol. 2010;37(5):533-546.

4. Hodi FS, O’Day SJ, McDermott DF, et al. Improved survival with ipilimumab in patients with metastatic melanoma. $N$ Engl J Med. 2010;363(8):711-723.

5. Bristol-Myers Squibb. Yervoy (ipilimumab) [package insert]. Princeton, NJ: Bristol-Myers Squibb; 2011.

6. Agarwala SS. Current systemic therapy for metastatic melanoma. Expert Rev Anticancer Ther. 2009;9(5):587-595.

7. Robert C, Thomas L, Bondarenko I, et al. Ipilimumab plus dacarbazine for previously untreated metastatic melanoma. $N$ Engl J Med. 2011;364(26):2517-2526.

8. Weber JS, Kähler KC, Hauschild A. Management of immune-related adverse events and kinetics of response with ipilimumab. J Clin Oncol. 2012;30(21):2691-2697.

9. Bristol-Myers Squibb. Ipilimumab US prescribing information: risk evaluation and mitigation strategy (REMS). 2012. Available from: http://www.yervoy.com/hcp/rems.aspx. Accessed July 12, 2013.

10. Dunn GP, Old LJ, Schreiber RD. The immunobiology of cancer immunosurveillance and immunoediting. Immunity. 2004;21(2): $137-148$.

11. Melero I, Hervas-Stubbs S, Glennie M, Pardoll DM, Chen L. Immunostimulatory monoclonal antibodies for cancer therapy. Nat Rev Cancer. 2007;7(2):95-106.

12. Grosso JF, Jure-Kunkel MN. CTLA-4 blockade in tumor models: an overview of preclinical and translational research. Cancer Immun. 2013;13:5.

13. Boasberg P, Hamid O, O'Day S. Ipilimumab: unleashing the power of the immune system through CTLA-4 blockade. Semin Oncol. 2010;37(5):440-449.

14. Ledezma B. Atypical clinical responses to immunotherapy in patients with advanced melanoma. Poster presented at: Oncology Nursing Society 36th Annual Congress; April 28-May 1, 2011; Boston, MA.

15. Wolchok JD, Neyns B, Linette G, et al. Ipilimumab monotherapy in patients with pretreated, advanced melanoma: a randomised, doubleblind, multicentre, phase 2, dose-ranging study. Lancet Oncol. 2010; 11(2):155-164.

16. Bristol-Myers Squibb. Phase 3 trial in subjects with metastatic melanoma comparing $3 \mathrm{mg} / \mathrm{kg}$ ipilimumab versus $10 \mathrm{mg} / \mathrm{kg}$ ipilimumab. Available from: http://clinicaltrials.gov/ct2/show/NCT01515189. NLM identifier: NCT01515189. Accessed July 12, 2013.

17. Bhardwaj N. Harnessing the immune system to treat cancer. J Clin Invest. 2007;117(5):1130-1136.

18. Robert C, Schadendorf D, Messina M, Hodi FS, O’Day S. Efficacy and safety of retreatment with ipilimumab in patients with pretreated advanced melanoma who progressed after initially achieving disease control. Clin Cancer Res. 2013;19(8):2232-2239.

19. National Comprehensive Cancer Network. NCCN guidelines version 2. 2013 updates - melanoma. 2012. Available from: http://www. nccn.org/professionals/physician_gls/pdf/melanoma.pdf. Accessed July 12, 2013.

20. Bristol-Myers Squibb. Study to compare the effect of ipilimumab retreatment with chemotherapy in advanced melanoma. Available from: http://www.clinicaltrials.gov/ct2/show/NCT01709162. NLM identifier: NCT01709162. Accessed July 12, 2013.

21. Lebbe C, Weber JS, Maio M, et al. Five-year survival rates for patients (pts) with metastatic melanoma (MM) treated with ipilimumab (IPI) in phase II trials. Poster presented at: 37th European Society of Medical Oncology (ESMO) Congress; September 28-October 2, 2012; Vienna, Austria.

22. Dougan M, Dranoff G. The immune response to tumors. Curr Protoc Immunol. 2009; Chapter 20:Unit 20.11.

23. Wolchok JD, Hoos A, O'Day S, et al. Guidelines for the evaluation of immune therapy activity in solid tumors: immune-related response criteria. Clin Cancer Res. 2009;15(23):7412-7420. 
24. Hoos A, Eggermont AM, Janetzki S, et al. Improved endpoints for cancer immunotherapy trials. J Natl Cancer Inst. 2010;102(18):1388-1397.

25. Ibrahim RA, Berman DM, de Pril V, et al. Ipilimumab safety profile: summary of findings from completed trials in advanced melanoma. J Clin Oncol. 2011;29 Suppl:8583.
26. Dummer R, Maio M, Hamid O, et al. Time to onset and resolution of immune-related adverse events associated with ipilimumab therapy in patients with advanced melanoma. Poster presented at: Perspectives in Melanoma XIV; September 17-18, 2010; Amsterdam, The Netherlands.

\section{Publish your work in this journal}

Cancer Management and Research is an international, peer-reviewed open access journal focusing on cancer research and the optimal use of preventative and integrated treatment interventions to achieve improved outcomes, enhanced survival and quality of life for the cancer patient. The journal welcomes original research, clinical \& epidemiological studies, reviews \& evaluations, guidelines, expert opinion \& commentary, case reports \& extended reports. The manuscript management system is completely online and includes a very quick and fair peerreview system, which is all easy to use. Visit http://www.dovepress.com/ testimonials.php to read real quotes from published authors. 